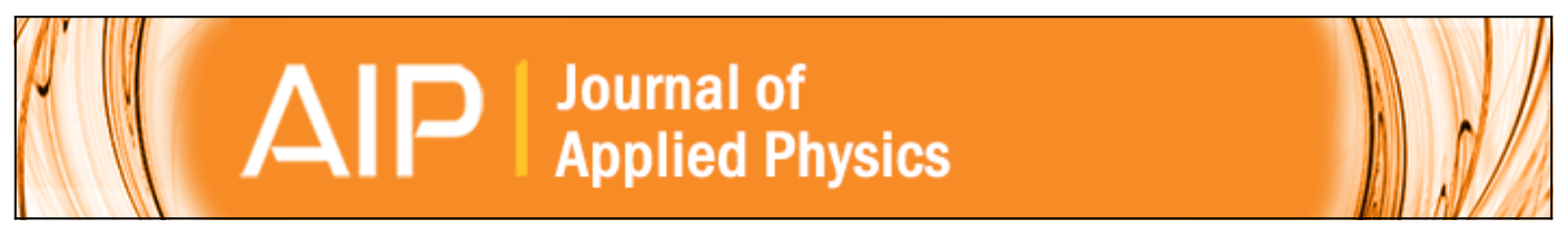

\title{
Development of FeNiMoB thin film materials for microfabricated magnetoelastic sensors
}

Cai Liang, Chinthaka Gooneratne, Dongkyu Cha, Long Chen, Yogesh Gianchandani, and Jurgen Kosel

Citation: Journal of Applied Physics 112, 113912 (2012); doi: 10.1063/1.4768458

View online: http://dx.doi.org/10.1063/1.4768458

View Table of Contents: http://scitation.aip.org/content/aip/journal/jap/112/11?ver=pdfcov

Published by the AIP Publishing

\section{Articles you may be interested in}

Development of magnetoresistive thin film sensor for magnetic field sensing applications

AIP Conf. Proc. 1512, 30 (2013); 10.1063/1.4790897

Microfabrication of magnetostrictive beams based on NiFe film doped with B and Mo for integrated sensor systems

J. Appl. Phys. 111, 07E515 (2012); 10.1063/1.3679016

Growth, structural, and magnetic characterizations of nanocrystalline $\mathrm{Y}^{\prime}$ - Fe Ni N ( 220 ) thin films

Appl. Phys. Lett. 90, 032505 (2007); 10.1063/1.2430920

Effect of heat treatment on microstructure and magnetic properties of anisotropic Nd-Fe-B films with Mo or Ti buffer layer

J. Appl. Phys. 98, 113905 (2005); 10.1063/1.2136208

Soft magnetic granular material Co-Fe-Hf-O for micromagnetic device applications

J. Appl. Phys. 97, 10 F907 (2005); 10.1063/1.1853238

MIT LINCOLN

LABORATORY CAREERS

Discover the satisfaction of innovation and service

to the nation
- Space Control

- Air \& Missile Defense

- Communications Systems \& Cyber Security

- Intelligence, Surveillance and

Reconnaissance Systems

- Advanced
Electronics
- Tactical Systems
- Homeland
Protection
- Air Traffic Control

LINCOLN LABORATORY

MassachusetTs Institute of TeChNOLOGY

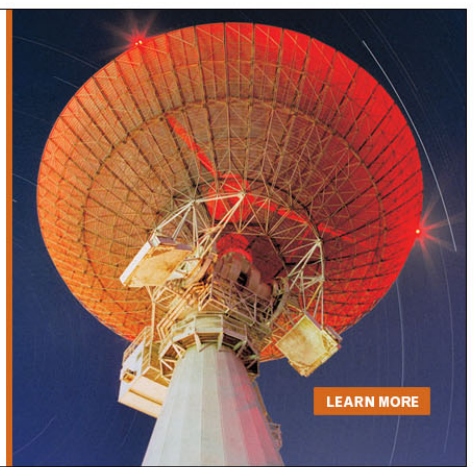




\title{
Development of FeNiMoB thin film materials for microfabricated magnetoelastic sensors
}

\author{
Cai Liang, ${ }^{1, \text { a) }}$ Chinthaka Gooneratne,${ }^{1}$ Dongkyu Cha, ${ }^{1}$ Long Chen, ${ }^{1}$ Yogesh Gianchandani, ${ }^{2}$ \\ and Jurgen Kosel ${ }^{1}$ \\ ${ }^{1}$ Computer Electrical and Mathematical Sciences and Engineering, King Abdullah University of Science \\ and Technology, 4700 KAUST, Thuwal 23955, Saudi Arabia \\ ${ }^{2}$ Department of Electrical Engineering and Computer Science, 1301 Beal Ave., University of Michigan, \\ Ann Arbor, Michigan 48109, USA
}

(Received 30 July 2012; accepted 6 November 2012; published online 7 December 2012)

\begin{abstract}
Metglas $^{\mathrm{TM}} 2826 \mathrm{MB}$ foils of $25-30 \mu$ m thickness with the composition of $\mathrm{Fe}_{40} \mathrm{Ni}_{38} \mathrm{Mo}_{4} \mathrm{~B}_{18}$ have been used for magnetoelastic sensors in various applications over many years. This work is directed at the investigation of $\sim 3 \mu$ m thick iron-nickel-molybdenum-boron (FeNiMoB) thin films that are intended for integrated microsystems. The films are deposited on Si substrate by cosputtering of iron-nickel (FeNi), molybdenum (Mo), and boron (B) targets. The results show that dopants of Mo and B can significantly change the microstructure and magnetic properties of FeNi materials. When FeNi is doped with only Mo its crystal structure changes from polycrystalline to amorphous with the increase of dopant concentration; the transition point is found at about 10 at. \% of Mo content. A significant change in anisotropic magnetic properties of FeNi is also observed as the Mo dopant level increases. The coercivity of FeNi films doped with Mo decreases to a value less than one third of the value without dopant. Doping the FeNi with B together with Mo considerably decreases the value of coercivity and the out-of-plane magnetic anisotropy properties, and it also greatly changes the microstructure of the material. In addition, doping B to FeNiMo remarkably reduces the remanence of the material. The film material that is fabricated using an optimized process is magnetically as soft as amorphous Metglas ${ }^{\mathrm{TM}} 2826 \mathrm{MB}$ with a coercivity of less than $40 \mathrm{Am}^{-1}$. The findings of this study provide us a better understanding of the effects of the compositions and microstructure of FeNiMoB thin film materials on their magnetic properties. (C) 2012 American Institute of Physics. [http://dx.doi.org/10.1063/1.4768458]
\end{abstract}

\section{INTRODUCTION}

Transverse vibration of cantilevers has been used for mass sensing and applied in chemical and biological detection for many years. ${ }^{1-5}$ Similarly, freestanding beams made of ferromagnetic material as a sensor platform have been widely studied for detection of physical, chemical, and biochemical substances. ${ }^{6-13}$ These beams operate in the longitudinal mode offering a higher resonant frequencies compared to the transverse mode. Figs. 1(a) and 1(b) show a cantilever vibrating in the transverse and longitudinal mode, respectively, while Fig. 1(c) shows a freestanding beam vibrating in the longitudinal mode. The operation principle of such vibration-based sensors is to utilize a change in the resonant frequency as a result of the target molecules binding (thereby adding mass) on the sensors surface. The intrinsic mass sensitivity of such sensors is defined as the resonant frequency change per mass change, which is proportional to the resonant frequency and inversely proportional to the mass of the sensor. ${ }^{14}$ To increase the mass sensitivity, a reduction of the weight and increase of frequency of a sensor is essential. In particular, the reduction of the sensor length is an effective method as it not only increases the frequency but also

\footnotetext{
a) Author to whom correspondence should be addressed. Electronic mail: cai_liang@live.com.
}

decreases the weight of the sensor. ${ }^{15}$ Therefore, miniaturizing the sensor is an effective approach to improve its performance.

Commercially available Metglas $^{\mathrm{TM}} 2826 \mathrm{MB}$ strips ( $\sim 28 \mu \mathrm{m}$ thickness) have been utilized effectively as magnetoelastic sensor platforms because of two reasons (1) very soft magnetic properties, e.g., coercivity values less than 100 $\mathrm{Am}^{-1}$ and remanence values less than $3 \%$ requiring a relatively small magnetic field to magnetize the material; (2) an amorphous structure, which leads to a significant reduction of the magnetocrystalline anisotropy. As a result of these properties, sensors fabricated from Metglas material are easy to actuate and are highly sensitive to mass loads. ${ }^{13}$ Although fabricating a sensor from bulk scale Metglas strips in the range of $1 \mathrm{~mm}$ is possible with advanced machining techniques, thin film devices are needed for further miniaturization and integration with electronics. The motivation of this study is to fabricate a ferromagnetic FeNiMoB thin film material that can be used for fabricating sensor elements in the microscale range, in order to increase the mass sensitivity of the device. Previous studies employing co-sputtering of $\mathrm{Fe}$ and B targets or direct sputtering of Metglas ${ }^{\mathrm{TM}} 2826 \mathrm{MB}$ to produce $\mathrm{Fe}-\mathrm{B}$ and $\mathrm{FeNiMoB}$ films, respectively, for microscale magnetoelastic sensors have shown very promising results. ${ }^{16,17}$ In the present work, FeNi thin films are doped with Mo and B using co-sputtering of FeNi, Mo and B 

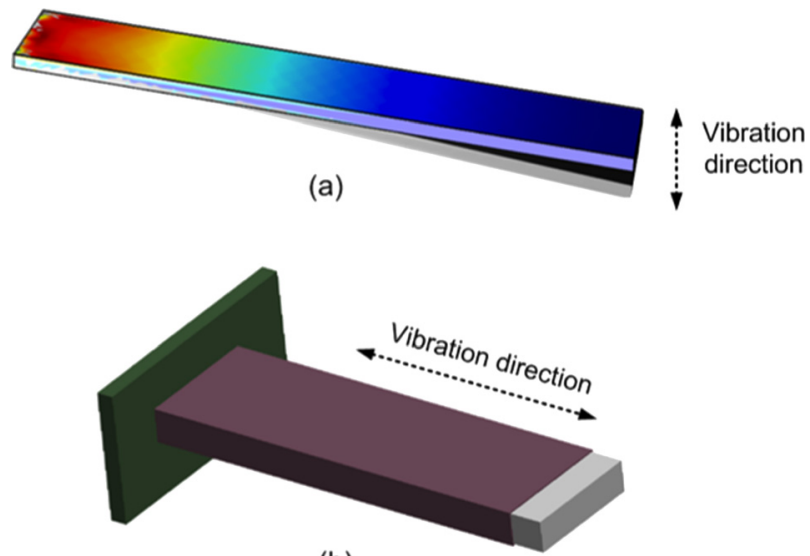

(b)

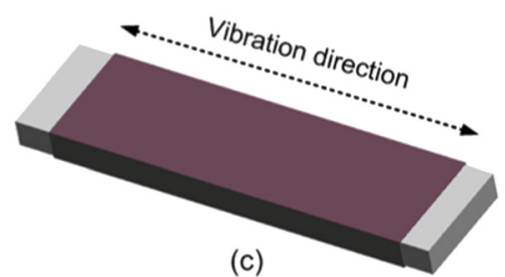

FIG. 1. (a) Cantilever vibrating in the transverse mode, (b) cantilever vibrating in the longitudinal mode, (c) freestanding beam vibrating in the longitudinal mode.

targets to study the materials' properties. Based on the optimized process parameters, freestanding elements with $500 \mu \mathrm{m}$ and $100 \mu \mathrm{m}$ lengths have been fabricated using conventional microfabrication technology.

\section{EXPERIMENTAL PROCEDURE}

FeNi-based thin film materials were deposited on a silicon (100) substrate via co-sputtering FeNi, Mo, and B targets using PVD 75, a three-source sputter system from Lesker Inc. All targets were $75 \mathrm{~mm}$ in diameter and had a purity of 99.99\% for the respective elements. The atomic concentration of $\mathrm{Fe}$ and $\mathrm{Ni}$ in the FeNi target was designed to be 50:50. DC power was chosen for sputtering of all the targets. The distance between the substrate and targets was approximately $16.5 \mathrm{~cm}$. The base pressure of the chamber was
$3 \times 10^{-7}$ Torr or lower, while the sputtering pressure was set to $7 \mathrm{mTorr}$ of Ar. Before each deposition process, all targets were sputter-cleaned for $10 \mathrm{~min}$ with the shutter closed to remove any contaminants or oxides. A strong magnetron target holder was used for FeNi to compensate for the sputter yield reduction due to the magnetic property of the target material. The substrate holder was rotated at a speed of $20 \mathrm{rpm}$ during the deposition process. Table I lists the details of the sputtering parameters that were used for the thin film deposition. The film thickness was measured using Dektak $6 \mathrm{M}$ stylus profiler.

A Nova NanoSEM (FEI, Netherlands) scanning electron microscope (SEM) equipped with an energy-dispersive $\mathrm{X}$-ray Spectroscopy (EDS) detector was used to characterize the morphology, elements distribution and composition of the films. X-ray photoelectron spectroscopy (XPS) measurements were carried out, using a Kratos Axis Ultra DLD system from Kratos Analytical, for analyses of the composition of the films. High-resolution spectrums were taken after the samples were etched for $300 \mathrm{~s}$ to reduce oxidation and contamination effects. A Rigaku Rotaflex x-ray diffractometer (XRD) with $\mathrm{Cu}-\mathrm{K} \alpha 1$ radiation and FEI Titan Super-Twin transmission electron microscopy (TEM) were used to characterize the crystal structure of the films. TEM samples were prepared by using Helios 400s (FEI, Netherlands) focused ion beam (FIB) equipped with a nanomanipulator. After attaching the sample to the $\mathrm{Cu}$ TEM grid with lift-out method, the sample was cleaned using a low energy Ga ion beam to get rid of damaged areas. High-resolution TEM imaging and selected area diffraction (SAD) was performed on each sample.

Magnetic properties were studied with a vibrating sample magnetometer (VSM) model 1660 and a magnetic force microscope (MFM) model 5400 SPM from Agilent Technology. In-plane VSM measurements in two directions perpendicular to each other were conducted for each sample to study the in-plane magnetic anisotropic properties. Out-ofplane properties were characterized with the applied magnetic field perpendicular to the film plane. Cobalt-coated MFM probes with spring constant of $2.8 \mathrm{~N} / \mathrm{m}$ and resonant frequency of $75 \mathrm{kHz}$ were used to study the magnetic domain

TABLE I. Sputter process conditions of samples and their properties. Hc: coercivity; Ms: saturation magnetization; Mr: remanence; $\mathrm{H}^{*}$ : the field $\mathrm{H}$ at $\mathrm{M}=75 \% \mathrm{Ms}, \mathrm{Ms}, \mathrm{Mr}$ and $\mathrm{H}^{*}$ are the in-plane magnetization data.

\begin{tabular}{|c|c|c|c|c|c|c|c|c|c|c|c|c|c|}
\hline \multirow[b]{2}{*}{ Sample } & \multicolumn{3}{|c|}{ Sputtering power (w) } & \multirow{2}{*}{$\begin{array}{l}\text { Thickness } \\
\text { (nm) }\end{array}$} & \multicolumn{4}{|c|}{ Composition (at. \%) } & \multicolumn{2}{|c|}{$\mathrm{Hc}(\mathrm{kA} / \mathrm{m})$} & \multirow{2}{*}{$\begin{array}{c}\mathrm{Ms} \\
(\mathrm{kA} / \mathrm{m})\end{array}$} & \multirow{2}{*}{$\begin{array}{c}\mathrm{Mr} / \mathrm{Ms} \\
(\%)\end{array}$} & \multirow{2}{*}{$\begin{array}{c}\mathrm{H}^{*} \\
(\mathrm{kA} / \mathrm{m})\end{array}$} \\
\hline & $\mathrm{FeNi}$ & Mo & B & & $\mathrm{Fe}$ & $\mathrm{Ni}$ & Mo & B & In-plane & Out-of-plane & & & \\
\hline S1 & 225 & 0 & 0 & 410 & 45.70 & 54.30 & 0 & 0 & 5.17 & 9.82 & 9.75 & 21.9 & 35.41 \\
\hline S2 & 225 & 17.5 & 0 & 400 & 41.03 & 54.39 & 4.58 & 0 & 3.58 & 8.83 & 9.31 & 16 & 33.42 \\
\hline S3 & 225 & 20 & 0 & 422 & 42.96 & 51.80 & 5.24 & 0 & 3.30 & 10.66 & 7.10 & 26 & 21.25 \\
\hline S4 & 225 & 28 & 0 & 435 & 42.04 & 50.64 & 7.32 & 0 & 3.05 & 8.59 & 4.90 & 38.3 & 11.46 \\
\hline S5 & 225 & 35 & 0 & 416 & 41.99 & 48.69 & 9.32 & 0 & 2.86 & 15.36 & 6.94 & 49.2 & 7.96 \\
\hline S6 & 225 & 50 & 0 & 600 & 39.63 & 45.42 & 14.49 & 0 & 1.53 & 11.04 & 2.98 & 45.6 & 7.16 \\
\hline S7 & 200 & 20 & 75 & 650 & & & & & 0.62 & 2.47 & 3.49 & 8.9 & 18.78 \\
\hline S8 & 200 & 20 & 100 & 550 & & & & & 0.59 & 6.64 & 3.36 & 14 & 8.59 \\
\hline S9 & 200 & 35 & 100 & 570 & & & & & 0.22 & 3.28 & 3.65 & 15.8 & 3.50 \\
\hline S10 & 200 & 35 & 150 & 2700 & 38.83 & 37.48 & 10.25 & 13.52 & 0.04 & 0.52 & 0.41 & 4.3 & 1.11 \\
\hline S11 & \multicolumn{3}{|c|}{ Metglas $2826 \mathrm{MB}$} & 2800 & 40 & 38 & 4 & 18 & 0.075 & 0.071 & 0.45 & 2.9 & 2.31 \\
\hline
\end{tabular}




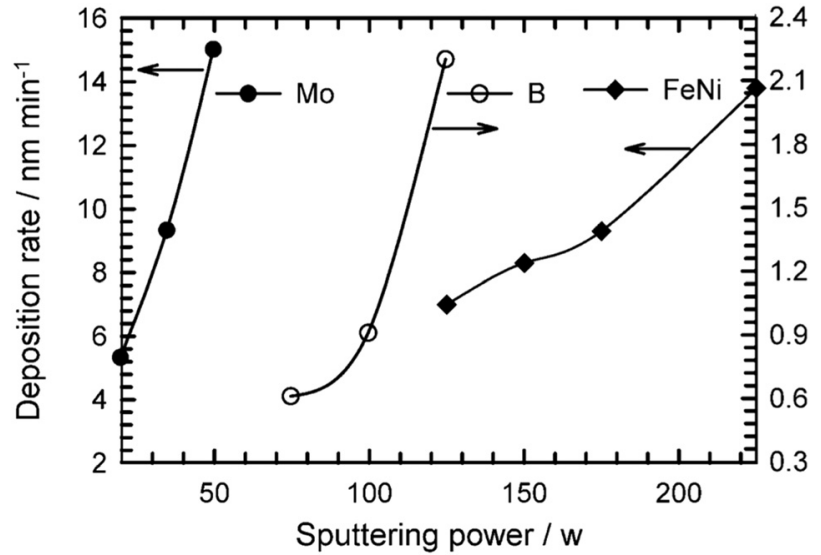

FIG. 2. Deposition rates of Mo, B and FeNi at different sputtering power (target diameter $75 \mathrm{~mm}$ ).

distribution. During MFM tests, the distance between the tip and the sample surface was kept at $200 \mathrm{~nm}$ to make sure the long-range dipolar magnetic force was dominant over Van der Waals force.

\section{RESULTS AND DISCUSSION}

\section{A. Synthesis of FeNiMoB thin films}

In order to fabricate a thin film material that has similar properties as Metglas ${ }^{\mathrm{TM}} 2826 \mathrm{MB}$, the film should have similar composition and structure as the Metglas, which has a composition of $\mathrm{Fe}_{40} \mathrm{Ni}_{38} \mathrm{Mo}_{4} \mathrm{~B}_{18}$ and an amorphous structure. Taking into account the atomic composition of Metglas ${ }^{\mathrm{TM}}$ $2826 \mathrm{MB}$ and the physical properties, e.g., density of $\mathrm{Fe}, \mathrm{Ni}$, $\mathrm{Mo}$, and $\mathrm{B}$ that are identical in both the bulk and thin film forms, and assuming the ratio of $\mathrm{Fe}$ to $\mathrm{Ni}$ to be close to 1 , the weight ratio of FeNi to Mo and B in Metglas ${ }^{\mathrm{TM}} 2826 \mathrm{MB}$ is calculated to be 5.97 and 11.77 , respectively. ${ }^{18}$ To produce a thin film of FeNiMoB with such composition by cosputtering, the ratios of the deposition rates for FeNi to Mo and B targets should be 7.27 and 3.28, respectively.

Fig. 2 shows the deposition rates for three targets (target size: $75 \mathrm{~mm}$ diameter) at a pressure of $7 \mathrm{mTorr}$ under different sputtering power levels. It can be noted that the deposition rate of Mo is relatively high even at low power levels, while it is very low for B even at high power levels. To better understand the dopant effect in FeNiMoB materials, a set of experiments for deposition of FeNiMo without the presence of B was conducted first. Further experiments were then performed with the addition of $B$ to the film to achieve optimum deposition conditions with respect to the microstructure and magnetic properties.

\section{B. Microstructure characterization}

The thin films' surface morphology was examined using SEM. It was observed that the higher the sputtering power the rougher the surface. The surface morphology of all FeNi films also exhibited nano scale cracks (that are typically a few hundred nanometers long and a few nanometers wide) regardless of the sputtering power. The sputtering pressure (or the Ar flow rate) may affect the internal stress of the thin film. An earlier study showed that a sputtering process at a pressure ranging from 3 to 6 mTorr resulted in FeNiMoB thin films with nano cluster morphology, an amorphous structure and better magnetic properties. ${ }^{17}$ The sputtering pressure was 7 mTorr in this study because the plasma could not be ignited when the pressure was lower than 6.5 mTorr. Fig. 3 shows SEM images of thin films deposited under the conditions listed in Table I. When FeNi was doped with various concentrations of Mo, the nano-scale cracks reduced and the films became smoother. From the images S1-S6, one can see that both FeNi and FeNiMo films developed nano-scale granularity with a similar growing mechanism. The surface

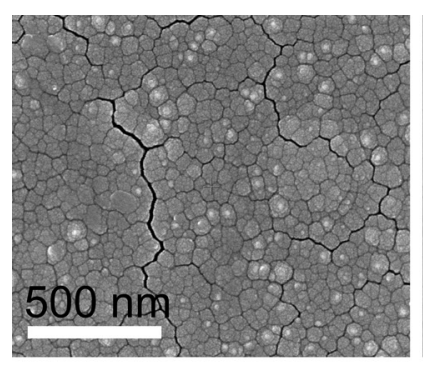

$\mathrm{S} 1: \mathrm{FeNi}$

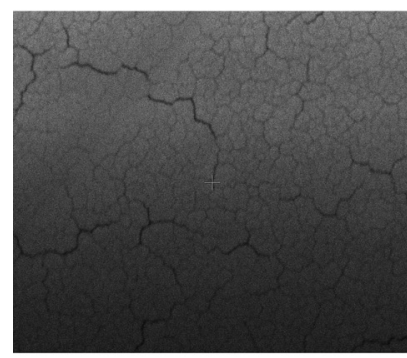

S6: FeNiMo

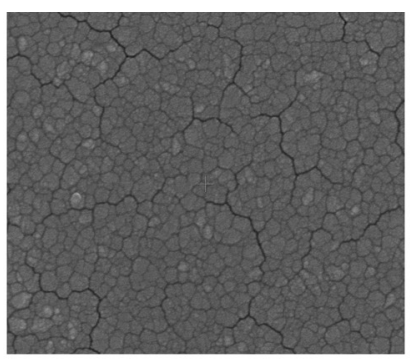

S2: FeNiMo

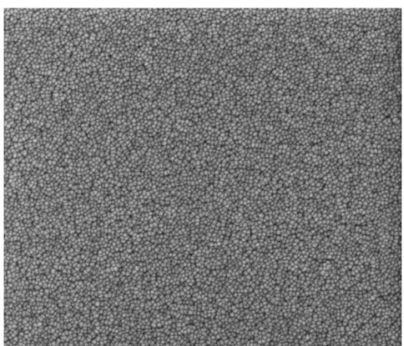

S7: FeNiMoB

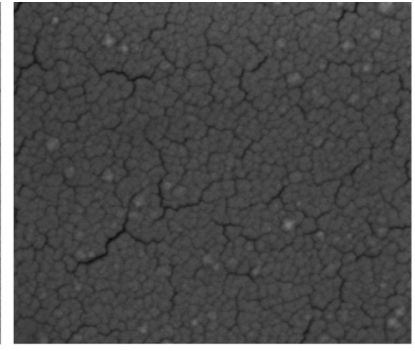

S4: FeNiMo

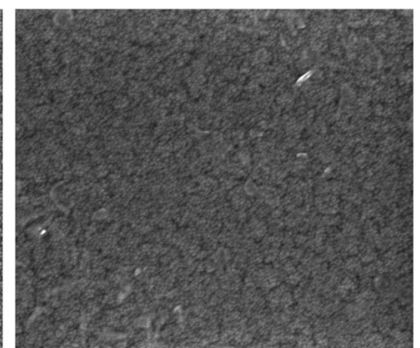

S9: FeNiMoB

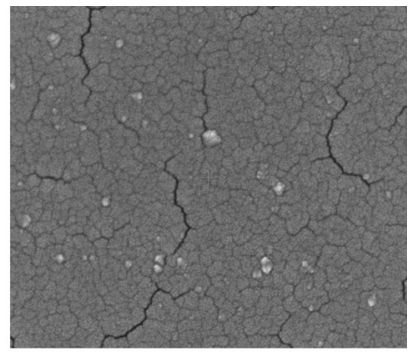

S5: FeNiMo

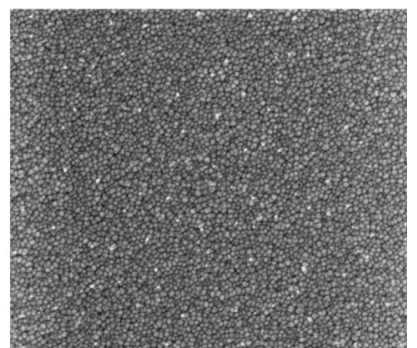

S10: FeNiMoB

FIG. 3. SEM images of FeNi films with different dopants as listed in Table I. All images have the same scale bar as shown in S1. 


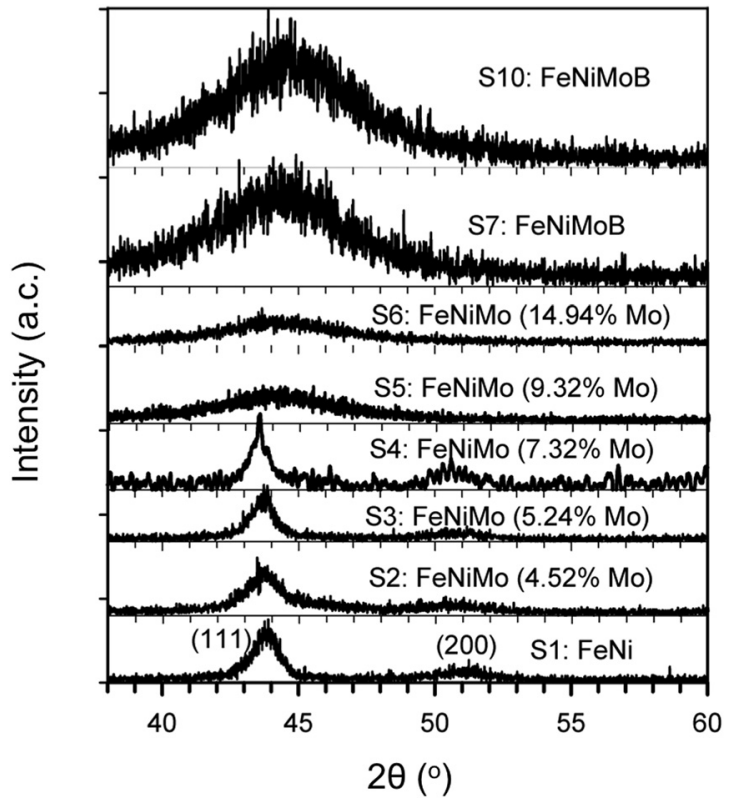

FIG. 4. XRD spectra for FeNi films with different dopants and concentrations. Note that the spectra for S7 and S10 have a higher resolution diffraction setting.

morphology became much smoother and the nano scale cracks in the films were completely eliminated, when the FeNi films were doped with both Mo and B elements, as seen in Fig. 3, images S7, S9, and S10. The elemental mapping and the composition of FeNi and FeNiMo films were analyzed by EDS; FeNiMoB was analyzed using an XPS as it has better resolution for B analysis. Due to the restricted access to XPS, only sample S10 was analyzed. It was observed that the distribution of elements in all films was even. The results of the atomic compositions are listed in Table I. In summary, the atomic ratio of $\mathrm{Fe}$ to $\mathrm{Ni}$ in films is less than 1 for samples S1 to S6 even though it was designed to be 1 in the sputter target. This discrepancy has been reported in the literature before ${ }^{19}$ and may be due to (a) the analytical error from EDS (usually it has a $3 \%$ variation); (b) the actual amount of $\mathrm{Fe}$ and $\mathrm{Ni}$ in the FeNi target not being equal; (c) the sputtering process not being in the equilibrium state, at which the film materials would have the same composition of the target, particularly for the case of sputtering alloy. ${ }^{20}$

Fig. 4 shows the XRD spectra of film materials deposited under the sputtering conditions listed in Table I. A facecentered cubic (FCC) polycrystalline structure of FeNi films (sample S1) was characterized by the presence of peaks from (111), (200), and (220) planes since the content of Fe is less than $60 \%$ in the Fe-Ni phase. This finding corresponds with the previously published reports. ${ }^{21}$ Note that the peak of (220) is not plotted due to the high intensity of the silicon peak present at $2 \theta$ near $69^{\circ}$. When FeNi was doped with Mo, the structure of the film started to change from polycrystalline to amorphous. The diffraction intensity at the (200) plane gradually decreased, and the spectral peaks disappeared while leaving a broad one at the (111) plane, when sputtering power of Mo increased to $35 \mathrm{~W}$. The corresponding dopant concentration of Mo was 9.32 at. \% or higher. In addition, it can be seen that the peak at the (111) position is slightly shifted towards a lower angle, in case of the crystalline FeNiMo films with low Mo content. This diffraction angle shift is due to the change in distance between (111) planes, which is an evidence of stress in the film materials. The amorphous structure yielded a film without nano cracks and with a smooth morphology. When both Mo and B were incorporated into FeNi films, the structure became amorphous, showing a broad diffraction spectrum as can be seen for samples S7 and S10 in Fig. 4. Note that higher diffraction resolution settings were used in those cases. The lattice constant and the grain size were calculated based on the XRD profile of (111) plane. Their values change with the Mo content as shown in Fig. 5, and they are approximately $3.58 \AA$ and $80 \AA$, respectively. The lattice constant also agreed well with the values reported previously. ${ }^{21,22}$ In the range of higher Mo content, the corresponding lattice constant and grain size are about $3.55 \AA$ and $22 \AA$, respectively. However, these values are only rough estimates. No information about the lattice and grain size was extracted from the XRD spectrum for FeNiMoB films, since they are amorphous in structure.

Additional investigations of the microstructure for selected FeNi, FeNiMo and FeNiMoB films were performed using TEM. No nano-scale cracks were found in the cross sectional TEM images, although such cracks were visible on the surface of some films in the SEM images (Fig. 3). The high-resolution TEM images, with the SAD insets, are shown in Fig. 6. A continuous multiple-ring type SAD pattern was seen in FeNi without dopant (S1) and with a small amount of Mo (5.24 at. \%) dopant (S3), which indicates a polycrystalline material with nano grains. When the concentration of Mo increased to 9.32 at. \% (S5), the SAD pattern changed to a single-ring type. This suggests only a single (111) phase existing in the material. Further increasing the Mo concentration to 14.94 at. \% (S6) resulted in the SAD pattern becoming a halo ring, which indicates amorphous film materials with a very small grain size. When FeNi was doped with both Mo and B (S9, S10), the SAD patterns became a broadened-ring type implying a completely amorphous structure evolving in these materials. HRTEM images in Fig. 6 confirm that a nano-scale, polycrystalline structure of Fe-Ni phase has evolved in S1, S3, and S5. Progressively less crystallinity in FeNiMo was seen in S3 and S5, when the

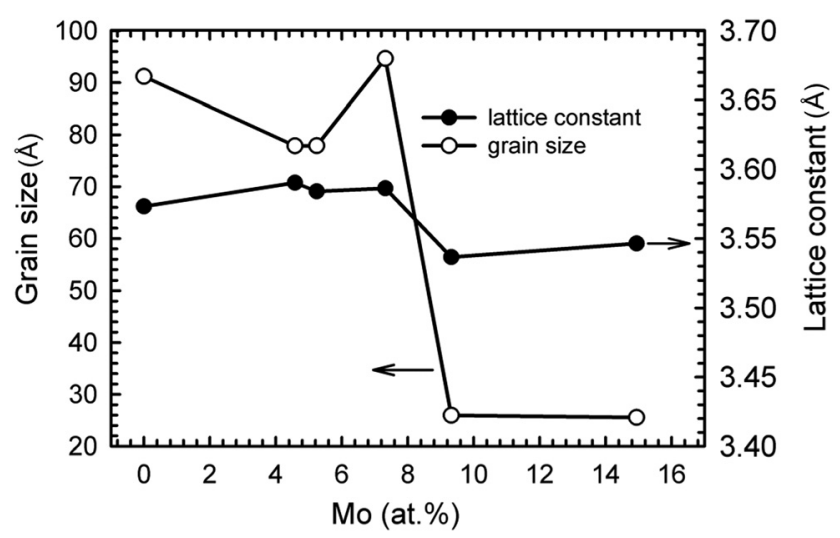

FIG. 5. Grain size and lattice constant of FeNi films as a function of dopant (Mo) concentration. 


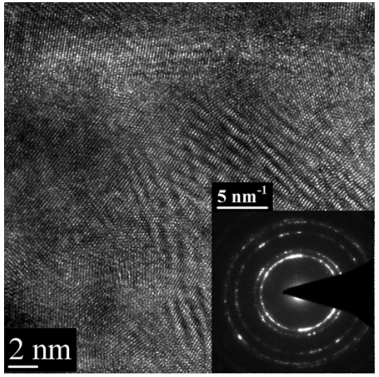

S1

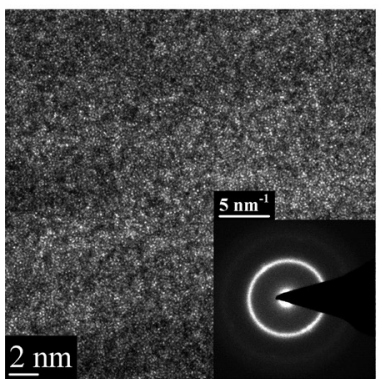

S6

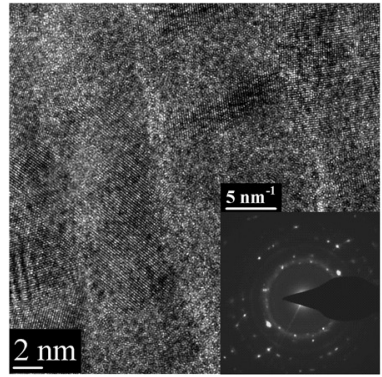

S3

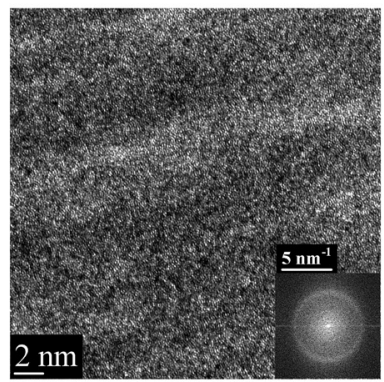

S9

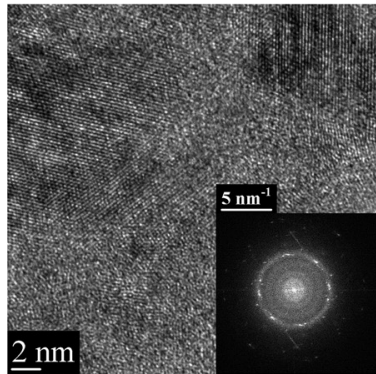

S5

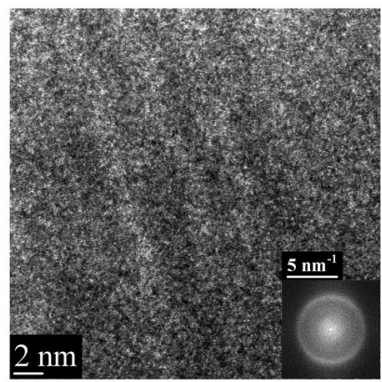

S10
FIG. 6. TEM images with SAD inset for FeNi films with different dopants as listed in Table I. Polycrystalline with various gain size can be seen in the images of $\mathrm{S} 1, \mathrm{~S} 3$ and $\mathrm{S} 5$, and no grain can be defined in the images of S6, S9 and S10. dopant of Mo content increased to 5.24 and 9.32 at. \%, respectively. With high Mo content (S6), grains were barely distinguishable. Additionally, no grains were seen in FeNiMoB films (S9 and S10). These findings by TEM are consistent with the XRD results except for S5. Sample S5 was characterized to FeNiMoB films (S9 and S10). These findings by TEM are consistent with the XRD results except for S5. Sample S5 was characterized to be amorphous in structure using XRD, shown by the broad and barely visible peak at the (111) position. However, it was characterized as crystalline using SAD and HRTEM. A FeNiMo film with a slightly higher Mo content (11.24 at. \%) was prepared to verify the Mo content at which the phase transition occurs. This sample was characterized as amorphous in structure by both XRD and TEM. A Mo content of $\sim 10$ at. $\%$ seems to be the maximum level that FeNi can incorporate without significantly changing its crystal structure. Banerjee and coworkers reported that an FeNiMo film with 13.5 at. \% Mo content was found to be of FCC structure. ${ }^{22}$ However, since the Fe content was only about 3.3 at. \% in their films, it is believed that the content of $\mathrm{Fe}$ and Mo was not high enough to disorder the Ni phase. In our case, FeNi with a higher dopant of Mo (S6 with 14.94 at. \% Mo), and with multiple dopants (S9 and S10 doped with both Mo and B at various levels) was amorphous in structure. Samples S9 and S10 revealed a fully amorphous phase in the films as the SAD patterns became blurry; particularly the first, broad halo ring.

\section{Magnetic properties of FeNiMoB films}

The magnetic properties were characterized using a VSM in both in-plane and out-of-plane directions. FeNi becomes magnetically softer when doped with Mo or Mo and B (see Table I). The coercivity and saturation magnetization decrease significantly with the increase of Mo dopant concentration, and a further considerable reduction occurs with dop- ing both Mo and B in the films. This effect of Mo dopant was also reported in earlier studies. ${ }^{19,22,23}$ A remarkable decrease of the field $\left(\mathrm{H}^{*}\right)$ required to magnetize the material to $75 \%$ of its saturation magnetization is also seen with increasing Mo level, which indicates the saturation is achieved easier. The remanence, however, increases in FeNiMo films with increasing Mo concentration, as seen by the change in the normalized magnetization (Mr/Ms). Adding B to the FeNiMo films has appreciably reduced the remanence. It was observed that the magnetic properties within the film plane were isotropic, which is due to the absence of a magnetic field applied and the continuous rotation of the substrate during the process of film deposition. Such in-plane magnetic isotropy was also observed by others in the FeNiMo thin films. ${ }^{19}$

Fig. 7 shows the magnetization loops for the in-plane and out-of-plane directions. To eliminate the volume effect normalized values are used for the analysis of the magnetization loops. Note that the magnetization loops for both inplane and out-of-plane directions are normalized using the total moments in the in-plane magnetization direction. During the VSM testing, a plastic holder was used to hold samples, in order to avoid any influence from the holder material. The film surface was set perpendicular to the applied field direction, when conducting the out-of-plane magnetization tests. The in-plane and out-of-plane magnetic properties have significantly changed with the dopants and their concentration. The film plane exhibits an easy axis of magnetization for all film materials.

Fig. 7(a) shows that with the concentration of Mo increasing, the magnetic properties become softer in the inplane directions, and the magnetic susceptibility of the films increases considerably. This is seen in the hysteresis loops that became increasingly steeper with increasing Mo content. In addition, the energy losses during the magnetization cycle have decreased. This indicates that the movement of domain walls in the film plane becomes easier. The out-of plane 
(a)

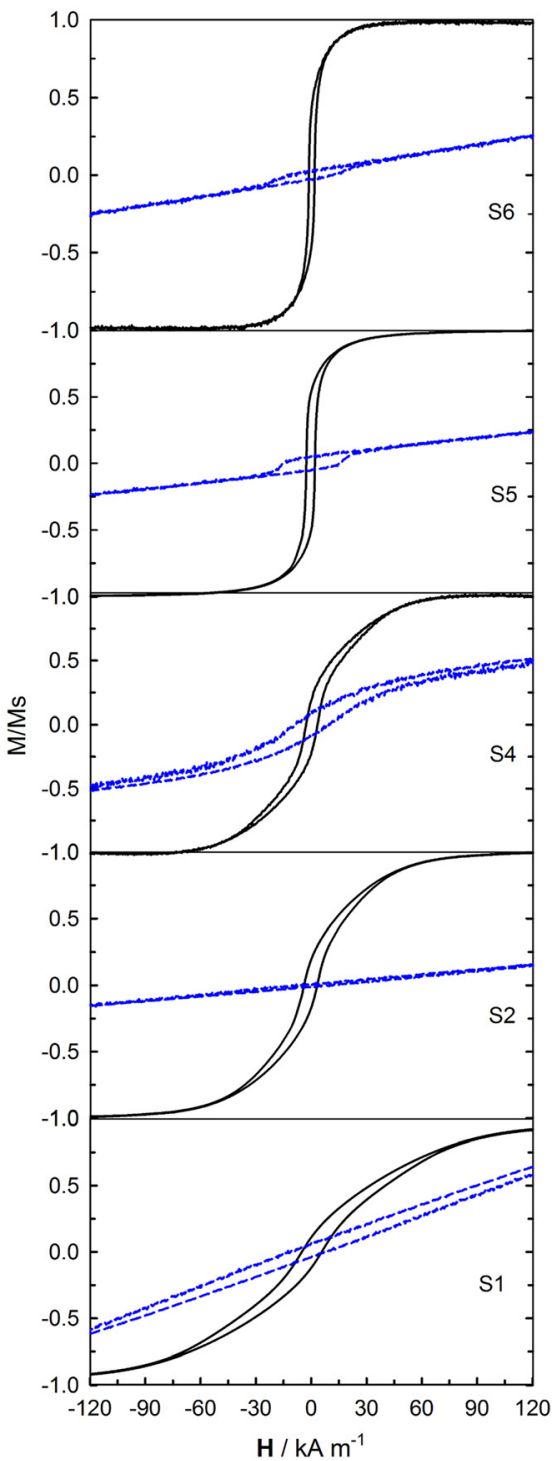

(b)

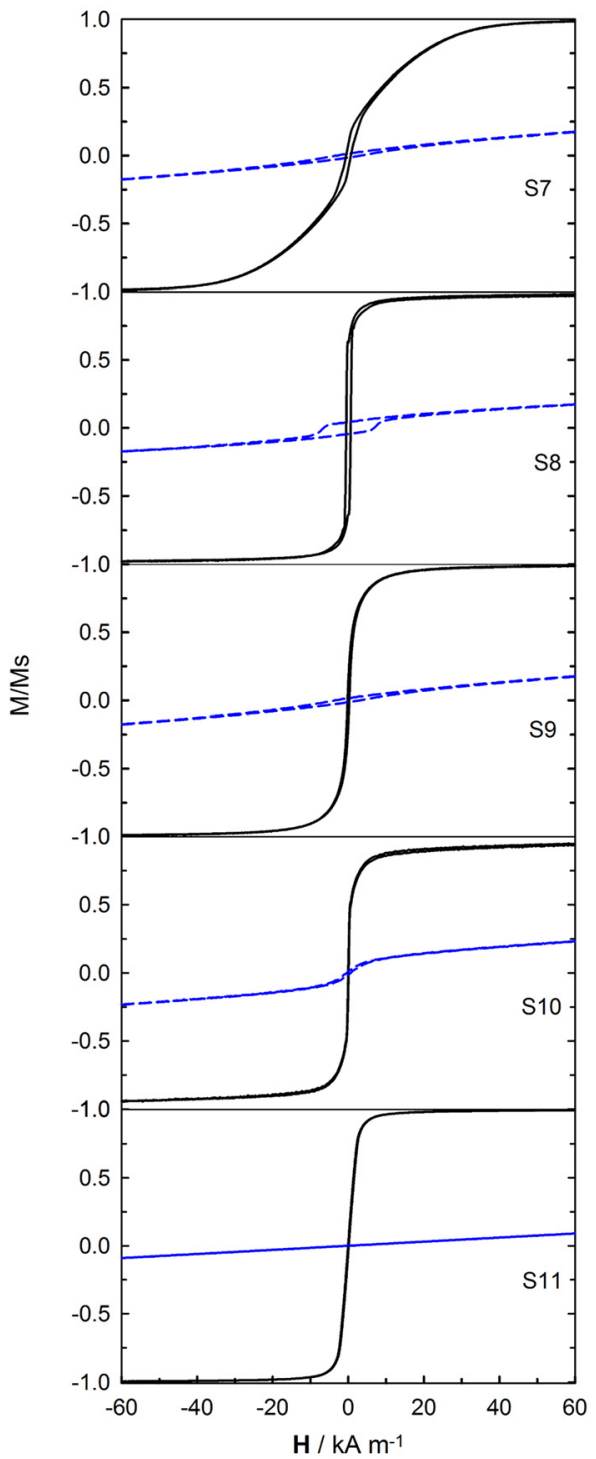

FIG. 7. Magnetization loops of FeNi films with various dopants conditions as listed in Table I. Black solid lines are the magnetization loops taken at the in-plane direction of the film, blue broken lines are the loops taken at the out-ofplane direction of the film. magnetization loops also significantly changed with the increase of Mo concentration. Such anisotropy changes can be explained by the crystal structure of the films. Samples S1 to $\mathrm{S} 4$ revealed FCC polycrystalline structures with (111) preferred orientation parallel to the film surface, as shown in Fig. 4. S5 was found to be polycrystalline from TEM examination but amorphous from XRD analysis. For a magnetic material with FCC crystal structure, the easy axis of magnetization is along the crystallographic direction of $\langle 111\rangle .^{24,25}$ Although the easy axis of magnetization is generally found in the film plane in a thin film material, the magnetocrystalline anisotropy favors the direction perpendicular to the film surface. Therefore, the deposited films with less crystallized structures are easier to magnetize along their surfaces. In this study the structure of FeNi film became less crystalline with increasing Mo content, and, as a result, the effect of magnetocrystalline anisotropy was reduced and the perpendicular anisotropy started to be broken down.

Fig. 7(b) shows the magnetization loops of films doped with both Mo and B under various conditions, and it can be seen that these films became even softer. These changes are most likely due to the fact that amorphous microstructures have evolved in FeNiMoB films (see Fig. 6 S6, S9, S10). An increase of either dopant level resulted in a softer material (see Table I). For instance, with the same sputtering conditions for $\mathrm{FeNi}$ and Mo targets, increasing the sputtering power for B resulted in a lower coercivity (S7 vs. S8, S9 vs. $\mathrm{S} 10)$, and the same is true in the case of fixed deposition conditions for FeNi and B targets and varying ones for Mo (S8 vs. S9). A significant change of magnetic properties was observed between samples S9 and S10, when the sputtering power for B increased by $50 \mathrm{~W}$. With the higher B content, the coercivity remarkably reduced to $18 \%$ from $220 \mathrm{Am}^{-1}$ to $40 \mathrm{Am}^{-1}$, and remanence reduced to about $27 \%$, from $15.8 \%$ to $4.3 \%$. The coercive force in the out-of-plane direction reduced to $28 \%$, from $3.28 \mathrm{kAm}^{-1}$ to $0.52 \mathrm{kAm}^{-1}$. This may be the reason why Metglas ${ }^{\mathrm{TM}} 2826 \mathrm{MB}$ has a relatively high B content (18 at. \%). The magnetic properties of film S10 are very similar to those of Metglas ${ }^{\mathrm{TM}} 2826 \mathrm{MB}$ (sample S11). Examining a thinner FeNiMoB film of $600 \mathrm{~nm}$, deposited with the same conditions as used in sample S10, showed similar magnetic properties as sample S10, indicating that there was no thickness effect. Metglas ${ }^{\mathrm{TM}} 2826 \mathrm{MB}$ exhibits excellent soft properties, as can be seen from the hysteresis 


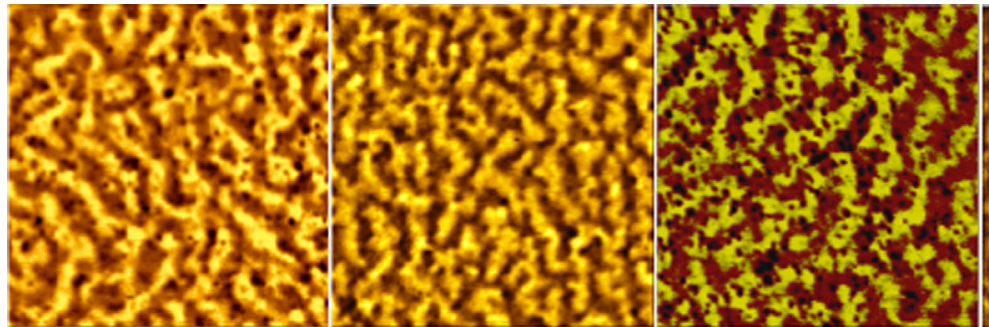

$\mathrm{S} 1: \mathrm{NiFe}$

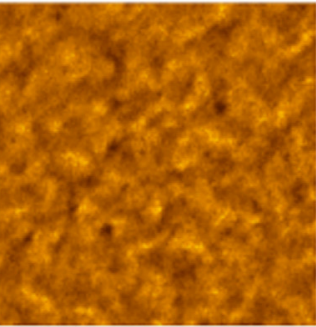

S6:NiFeMo
S2: NiFeMo

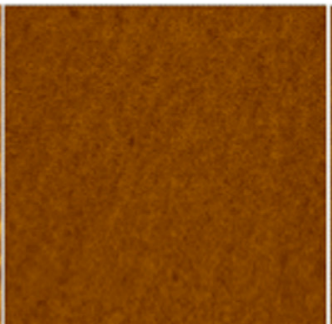

S7:NiFeMoB
S3: NiFeMo

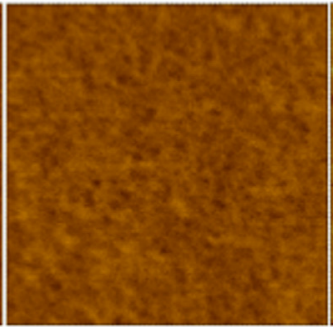

S8:NiFeMoB

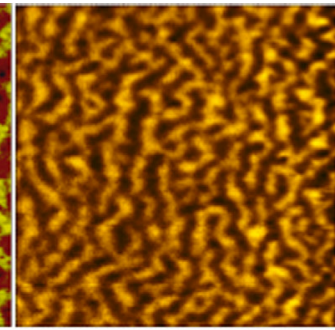

S4: NiFeMo

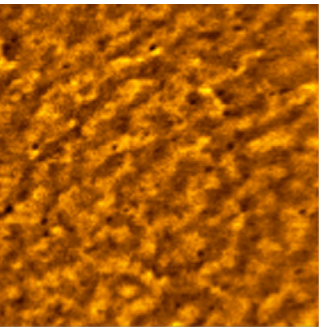

S5 NiFeMo

FIG. 8. MFM images ( long tails.

loop, i.e. (1) coercivity is as low as $70 \mathrm{Am}^{-1}$ in the easy directions; (2) perpendicular anisotropy is efficiently broken down, hence there is no domain wall movement in the thickness direction; (3) small remanence along the easy axis. Our sample S10 shows a small amount of domain wall displacement in the hard axis and a slightly higher remanence within the film plane, which probably results from the difference of composition between the two materials. The dopant composition in our deposited film has more Mo and less B content, which may not be sufficient enough to breakdown the perpendicular anisotropy in magnetic properties.

Fig. 8 shows MFM images of films deposited at various conditions. The MFM images were set at the same phase range $\left(10^{\circ}\right)$, so that they are compatible with the color contrast. As can be seen from the decreasing color contrast, the out-of-plane anisotropy component becomes smaller with the increase of Mo content from 0 to 14.94 at. \% (S1-S6). When doped with Mo and $\mathrm{B}$, the domain features were barely distinguishable in the out-of-plane direction (S 7 to S10). In order to ensure the contrast was not caused by the surface morphology, an AFM image was taken and analyzed simultaneously on the same area, while conducting the MFM measurement. This confirmed that the MFM images were not the reflection of the surface roughness. This observation agrees well with the magnetization curves as shown in Fig. 7. The VSM curves and MFM images show clear evidence that doping Mo and B to FeNi have significantly broken down the perpendicular anisotropy. Similar observations have been reported in the study of $\mathrm{FeNi} / \mathrm{Mo}$ multilayered film materials, where the use of Mo breaks down the perpendicular anisotropic magnetic properties. ${ }^{26}$

\section{Microfabrication of freestanding beams from FeNiMoB films}

Freestanding rectangular sensor platforms were fabricated, using standard microfabrication technology and the process parameters for sample S10. The process started with spin coating a thick photoresist on a Si substrate to generate rectangular templates. The magnetic film was then deposited on top. A lift-off process completed the fabrication of the freestanding sensors. Fig. 9 shows the SEM images of as fabricated
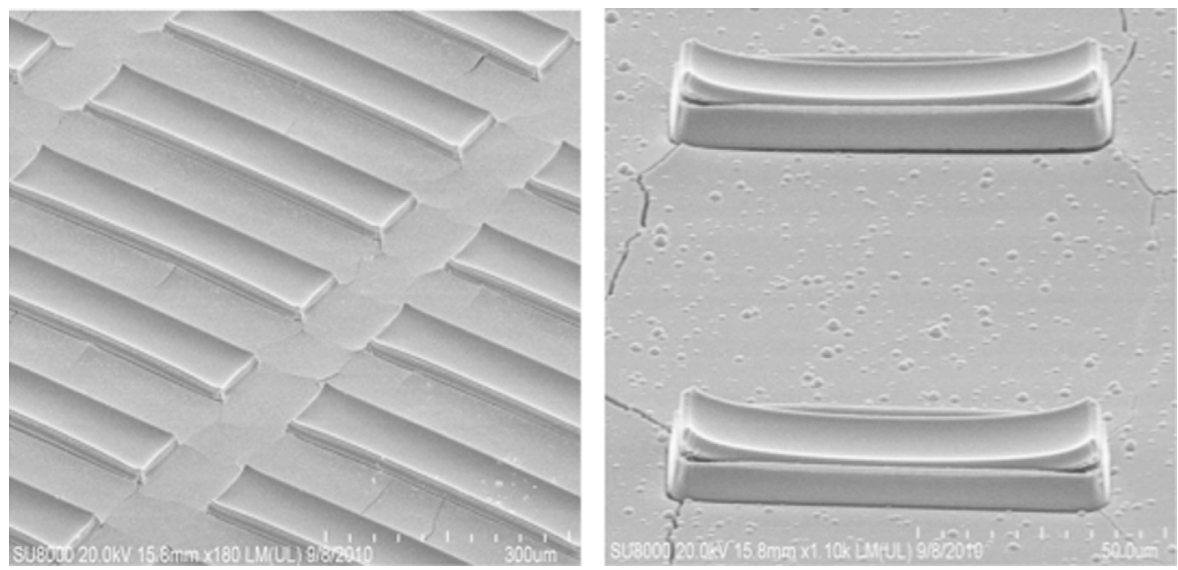

FIG. 9. SEM images of as fabricated freestanding beam sensor elements before lift-off. Beams with $500 \times 100 \mu \mathrm{m}$ and $100 \times 20 \mu \mathrm{m}$ are shown on the left and right hand side, respectively. 
rectangular elements on photoresist templates before lift-off. Some thermal stress developed in the beams due to the different coefficient of thermal expansion between the photoresist and magnetic film. This stress can be reduced or released by either controlling the temperature of the substrate during sputtering or annealing the beams in a vacuum chamber at about $215^{\circ} \mathrm{C}^{27}$

\section{CONCLUSIONS}

Ferromagnetic FeNi-based thin films have been deposited using a co-sputtering process. Crystallinity of FeNi ( 50:50) films doped with Mo decreased with the increase of Mo content, whereby about 10 at. \% of Mo was found to be the maximum value that FeNi can incorporate without a change of its crystalline structure. Mo doped to FeNi considerably softened the magnetic properties of films, due to the breakdown of the perpendicular anisotropy, but it also increased the remanence. FeNi films doped with both Mo and B exhibited an amorphous structure with nano clusters. Addition of B to FeNiMo not only further reduced the perpendicular anisotropy, which resulted in small coercive fields and hysteresis losses, but also significantly reduced the remanence. A coercivity as low as $40 \mathrm{Am}^{-1}$ was achieved with relatively high Mo dopant concentrations in FeNiMoB films, which is similar to Metglas 2826MB. Micro-scale freestanding magnetoelastic beams were successfully fabricated for future sensor applications.

\section{ACKNOWLEDGMENTS}

The authors gratefully thank the research group of Professor Rainer Schad at the University of Alabama for help with some of the VSM characterization. Thank is also due to Mr. QingXiao Wang and Dr. Ali Behzad for the help on SEM/EDS experiments. Authors acknowledge the fruitful discussion with Dr. Wenbo Mi. This work was performed in part at the Lurie Nanofabrication Facility at the University of Michigan. The Global Collaborative Research program at the King Abdullah University of Science and Technology (KAUST) sponsored this project.
${ }^{1}$ T. Thundat, E. A. Wachter, S. L. Sharp, and R. J. Warmack, Appl. Phys, Lett. 66, 1695 (1995).

${ }^{2}$ A. Passian, G. Muralidharan, A. Mehta, H. Simpson, T. L. Ferrell and T. Thundat, Ultramicroscopy 97, 391 (2003).

${ }^{3}$ A. Wig, E. T. Arakawa, A. Passian, T. L. Ferrell, and T. Thundat, Sens. Actuators B-Chem. 114, 206 (2006).

${ }^{4}$ B. Ilic, Y. Yang, K. Aubin, R. Reichenbach, S. Krylov, and H. G. Craighead, Nano Lett. 5, 925 (2005).

${ }^{5}$ G. A. Campbell and R. Mutharasan, Biosens. Bioelectron. 21, 597 (2005).

${ }^{6}$ S. Huang, H. Yang, R. S. Lakshmanan, M. L. Johnson, J. Wan, I.-H. Chen, H. C. Wikle III, V. A. Petrenko, J. M. Barbaree, and B. A. Chin, Biosens. Bioelectron 24, 1730 (2009).

${ }^{7}$ W. Shen, R. S. Lakshmanan, L. C. Mathison, V. A. Petrenko, and B. A. Chin, Sens. Actuators B 137, 501 (2009).

${ }^{8}$ R. Guntupalli, R. S. Lakshmanan, J. Hu, T. S. Huang, J. M. Barbaree, V. Vodyanoy, and B. A. Chin, J. Microbiol. Meth. 70, 112 (2007).

${ }^{9}$ C. A. Grimes, C. S. Mungle, K. Zeng, M. K. Jain, W. R. Dresche, M. Paulose, and K. G. Ong, Sensors 2, 294 (2002).

${ }^{10} \mathrm{~S}$. Li, Y. Li, H. Chen, A. Horikawa, W. Shen, A. Simonian, and B. A. Chin, Biosens. Bioelectron 26, 1313 (2010).

${ }^{11}$ M. Park, J. W. Park, H. C. Wikle III, and B. A. Chin, Sens. Actuators B (unpublished).

${ }^{12}$ S. R. Green, R. S. Kwon, G. H. Elta, and Y. B. Gianchandani, Biomed. Microdevices 12, 477 (2010).

${ }^{13}$ W. Shen, L. C. Mathison, V. A. Petrenko, and B. A. Chin, Appl. Phys. Lett. 96, 163502 (2010).

${ }^{14}$ B. Ilic, Y. Yang, and H. G. Craighead, Appl. Phys. Lett. 85, 2604 (2004).

${ }^{15}$ C. Liang, S. Morshed, and B. C. Prorok, Appl. Phys. Lett. 90, 221912 (2007).

${ }^{16}$ M. L. Johnson, O. LeVar, S. H. Yoon, J.-H. Park, S. Huang, D.-J. Kim, Z.-Y. Cheng, and B. A. Chin, Vacuum 83, 958 (2009).

${ }^{17}$ C. Liang, Ph.D. dissertation, Auburn University, USA, 2007.

${ }^{18}$ W. D. Callister, Jr. and D. G. Rethwisch, Fundamentals of Materials Science and Engineering: An Integrated Approach, 3rd ed. (John Wiley \& Sons, Asia, 2008).

${ }^{19}$ S. Cercelaru, A. M. Nguyen, P. Hesto, G. Tremblay, and J. C. Perron, J Magn. Magn. Mater. 160, 338 (1996).

${ }^{20}$ M. Ohring, Materials Science of Thin Films: Deposition and Structure, 2nd ed. (Academic, San Diego, 2002).

${ }^{21}$ X. G. Li, A. Chiba, and S. Takahashi, J. Magn. Magn. Mater. 170, 339 (1997).

${ }^{22}$ M. Banerjee, R. Banerjee, A. K. Majumdar, A. Mookerjee, B. Sanyal, and A. K. Nigam, Physcia B 405, 4287 (2010).

${ }^{23}$ A. M. Nguyen, S. Cercelaru, G. Tremblay, J. C. Perron, and P. Hesto, Thin Solid Films 275, 231 (1996).

${ }^{24}$ M. T. Johnson, P. J. H. Bloemen, F. J. A. d. Broeder, and J. J. d. Vries, Rep. Prog. Phys. 59, 1409 (1996).

${ }^{25}$ M. Farle, Rep. Prog. Phys. 61, 755 (1998).

${ }^{26}$ M. Romera, R. Ranchal, D. Ciudad, M. Maicas, and C. Aroca, J. Appl. Phys. 110, 083910 (2011).

${ }^{27}$ C. Liang, J. Hu, B. Prorok, C. Gooneratne, and J. Kosel, Mater. Sci. Forum 669, 1207 (2011). 